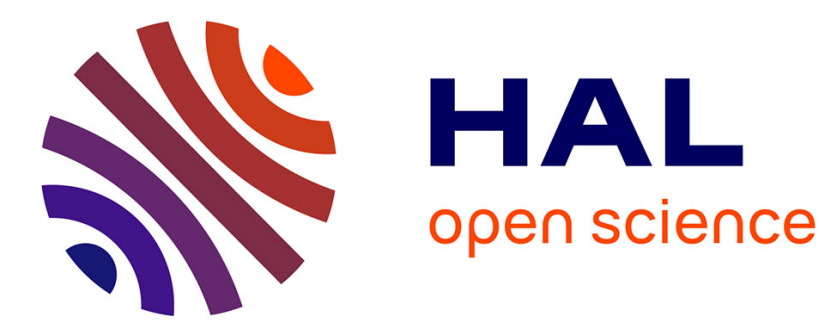

\title{
Front dynamics in fractional-order epidemic models
}

Emmanuel Hanert, Eva Schumacher, Eric Deleersnijder

\section{To cite this version:}

Emmanuel Hanert, Eva Schumacher, Eric Deleersnijder. Front dynamics in fractional-order epidemic models. Journal of Theoretical Biology, 2011, 279 (1), pp.9. 10.1016/j.jtbi.2011.03.012 . hal00694284

\section{HAL Id: hal-00694284 \\ https://hal.science/hal-00694284}

Submitted on 4 May 2012

HAL is a multi-disciplinary open access archive for the deposit and dissemination of scientific research documents, whether they are published or not. The documents may come from teaching and research institutions in France or abroad, or from public or private research centers.
L'archive ouverte pluridisciplinaire HAL, est destinée au dépôt et à la diffusion de documents scientifiques de niveau recherche, publiés ou non, émanant des établissements d'enseignement et de recherche français ou étrangers, des laboratoires publics ou privés. 


\section{Author's Accepted Manuscript}

Front dynamics in fractional-order epidemic models

Emmanuel Hanert, Eva Schumacher, Eric Deleersnijder

PII: $\quad$ S0022-5193(11)00152-4

DOI: $\quad$ doi:10.1016/j.jtbi.2011.03.012

Reference: $\quad$ YJTBI6405

To appear in: $\quad$ Journal of Theoretical Biology

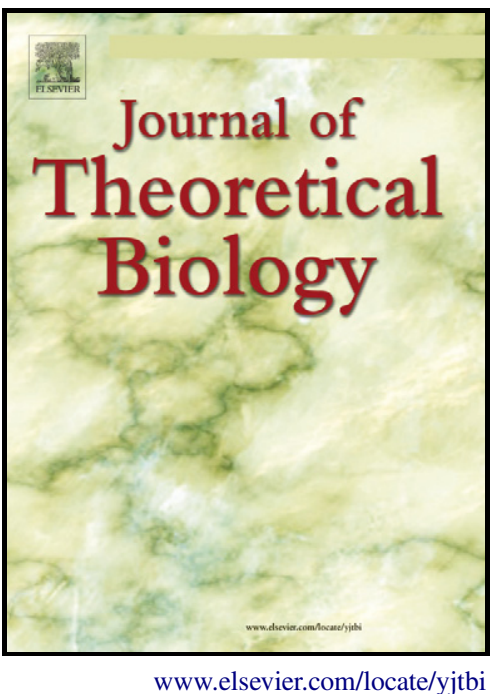

Received date: $\quad 7$ October 2010

Revised date: $\quad 21$ February 2011

Accepted date: $\quad 14$ March 2011

Cite this article as: Emmanuel Hanert, Eva Schumacher and Eric Deleersnijder, Front dynamics in fractional-order epidemic models, Journal of Theoretical Biology, doi:10.1016/j.jtbi.2011.03.012

This is a PDF file of an unedited manuscript that has been accepted for publication. As a service to our customers we are providing this early version of the manuscript. The manuscript will undergo copyediting, typesetting, and review of the resulting galley proof before it is published in its final citable form. Please note that during the production process errors may be discovered which could affect the content, and all legal disclaimers that apply to the journal pertain. 


\title{
Front dynamics in fractional-order epidemic models
}

\author{
Emmanuel Hanert ${ }^{\mathrm{a}, *}$, Eva Schumacher ${ }^{\mathrm{b}}$, Eric Deleersnijder ${ }^{\mathrm{b}, \mathrm{c}}$ \\ ${ }^{a}$ Université catholique de Louvain, Earth and Life Institute (ELI), Environmental \\ Sciences, Croix du Sud 2/16, B-1348 Louvain-la-Neuve, Belgium \\ ${ }^{b}$ Université catholique de Louvain, Institute of Mechanics, Materials and Civil \\ Engineering (iMMC), 4 Avenue G. Lemaître, B-1348 Louvain-la-Neuve, Belgium \\ ${ }^{c}$ Université catholique de Louvain, Earth and Life Institute (ELI), Georges Lemaître \\ Centre for Earth and Climate Research (TECLIM), 2 chemin du Cyclotron, B-1348 \\ Louvain-la-Neuve, Belgium
}

\begin{abstract}
A number of recent studies suggest that human and animal mobility patterns exhibit scale-free, Lévy-flight dynamics. However, current reaction-diffusion epidemics models do not account for the superdiffusive spread of modern epidemics due to Lévy flights. We have developed a SIR model to simulate the spatial spread of a hypothetical epidemic driven by long-range displacements in the infective and susceptible populations. The model has been obtained by replacing the second-order diffusion operator by a fractional-order operator. Theoretical developments and numerical simulations show that fractionalorder diffusion leads to an exponential acceleration of the epidemic's front and a power-law decay of the front's leading tail. Our results indicate the potential of fractional-order reaction-diffusion models to represent modern epidemics.
\end{abstract}

Keywords: Fractional-order diffusion, Lévy flights, epidemics spatial spread, front dynamics

\section{Introduction}

The spread of epidemics caused by directly-transmitted pathogens is re3 lated to the interactions between susceptible and infective individuals. The 4 occurrence of such interactions is a direct consequence of the mobility pat5 terns of individuals in their home range. Recent studies have shown that

\footnotetext{
*emmanuel.hanert@uclouvain.be
} 
mobility patterns for both humans and animals can be quite complex and exhibit a scale-free dynamics, characteristic of Lévy flights (see [3] for a review). Unlike ordinary Gaussian dispersion processes, Lévy flights are drawn from a probability distribution function with heavier tails than a Normal distribution. Such an asymptotic behaviour means that large displacements are more likely. Lévy flights are therefore superdiffusive as they disperse particles faster than a Gaussian random-walk. Lévy-flight patterns have been observed in the dispersion of bank notes [2], human mobility patterns derived from mobile phone data [18] as well as in the foraging patterns of a numbers of animal species $[39,40,36,23]$ although questions remain about the empirical evidence of some biological Lévy flights [15].

The existence of Lévy-flight mobility patterns suggests that modern epidemics cannot be represented by second-order reaction-diffusion models that implicitly assume a Gaussian dispersion process. Such models are only applicable when the infective and susceptible individuals travel short distances as compared to geographical distances. They lead to epidemic fronts that travel at a constant velocity and have been used, for instance, to model the spread of the Black Death in Europe in the 14th century [30]. Modern epidemics such as SARS or avian inflenza can spread around the world in a few weeks and seem to follow a non-Gaussian, scale-free dynamics [22, 37].

It has been shown that for Lévy-flight dispersion, the density function representing the population is the solution of a fractional-order diffusion equation $[6,28]$. Unlike integer-order derivatives that are local operators, fractional-order derivatives are non-local, integro-differential operators [31, 33]. As such, they can be used to represent memory effects and long-range dispersion processes. In the last decade, fractional-order diffusion models have been an active field of research both from a theoretical and applied perspective. They have been proposed to model a wide range of problems in surface and subsurface hydrology $[32,11,24]$, plasma turbulence $[9,10]$, finance $[35,27,19,4]$ and biology $[13,12]$.

In epidemiology, fractional-order models have just been mentionned in a couple of studies. Brockmann et al. [2] have shown that the density of bank notes originating from a given city is solution of a space-time fractional diffusion equation and have suggested that an epidemic spread could be modelled by a similar equation. In a subsequent study [1], Brockmann has proposed a SIR model that includes fractional-order diffusion. Some preliminary results are presented in that study but the properties of the model solutions are not discussed in detail. The goal of the present study is therefore to discuss in 
greater detail the properties of fractional-order epidemics model solutions. In particular, we consider the propagation of epidemic fronts and show that the use of a fractional-order diffusion term can lead to an acceleration of the front and thus a rapid spread of epidemics.

\section{Model description}

We shall consider a SIR model representing the spatial spread of an epidemic with non-local diffusion. The model takes into account 3 population densities, the susceptibles $S(x, t)$, the infectives $I(x, t)$ and a removed class $R(x, t)$. The spatial dynamics is assumed to be one-dimensional and is represented by a Riesz/Weyl, fractional-order differential operator denoted ${ }_{-\infty} D_{x}^{\alpha}$, where $1<\alpha \leq 2$ is the order of the spatial derivative. The fractional-order diffusivity is denoted $K_{\alpha}$ and has units of $\mathrm{m}^{\alpha} \mathrm{s}^{-1}$. The susceptible and infective populations are assumed to exhibit the same spatial dispersion patterns and are thus characterised by the same values of $\alpha$ and $K_{\alpha}$. The transmission from susceptibles to infectives and the disease-induced mortality are represented by a transmission-efficiency parameter $r$ and a mortality-rate parameter $a$, respectively. With these assumptions, the model equations read:

$$
\begin{aligned}
\frac{\partial S}{\partial t} & =-r I S+K_{\alpha}\left({ }_{-\infty} D_{x}^{\alpha} S\right) \\
\frac{\partial I}{\partial t} & =r I S-a I+K_{\alpha}\left({ }_{-\infty} D_{x}^{\alpha} I\right), \\
\frac{\partial R}{\partial t} & =a I
\end{aligned}
$$

where $r, K_{\alpha}$ and $a$ are positive constants. The dynamics of the removed class being entirely driven by the infectives and having no influence on the other two classes, it will not be further considered in the remainder of this study. The model equations have to be supplemented by a set of initial and boundary conditions to obtain a unique solution. The precise expression of the initial and boundary conditions is not required at this stage. We will just assume that the epidemic wave is advancing into a uniform population with an initially homogeneous susceptibles density $\mathcal{S}$.

The Riesz/Weyl fractional-order operator ${ }_{-\infty} D_{x}^{\alpha}$ is an integro-differential operator defined as follows:

$$
{ }_{-\infty} D_{x}^{\alpha} f(x)=\mathcal{F}_{k}^{-1}\left[(i k)^{\alpha} \hat{f}(k)\right]=\frac{1}{\Gamma(2-\alpha)} \frac{\partial^{2}}{\partial x^{2}} \int_{-\infty}^{x} \frac{f(y)}{(x-y)^{\alpha-1}} \mathrm{~d} y,
$$


where $\Gamma($.$) is Euler's gamma function and \mathcal{F}$ denotes the Fourier transform. When $\alpha=2$, the fractional-order derivative (4) reduces to a standard secondorder derivative. Eq. (4) defines a "left-sided" operator as it only takes into account the values of the function $f$ at the left-hand side of $x$. A right-sided operator (denoted ${ }_{x} D_{\infty}^{\alpha}$ ) can also be defined in a similar fashion (see for instance [33] for details). Combining left- and right-sided operators allows the definition of fractional-order derivative with arbitrary skewness. In this work, we only consider an asymmetric diffusion term in order to highlight the resulting different dynamics for left- and right-propagating epidemic waves.

The origin of anomalous, non-local diffusion lies in the random displacements of the individuals constituting the population. If one assumes that these individuals follow a Gaussian diffusive process, i.e. the distribution of random displacements has a finite variance, then the density of individuals is solution of a second-order diffusion equation. That result is a direct consequence of the central limit theorem (CLT). However, if one does not assume that the distribution of random displacements has a finite variance, the standard version of the CLT cannot be applied anymore. Instead, the generalization due to Lévy-Gnedenko $[17,25]$ can be used. That version of the theorem does not rely on the assumption that the sum of all the fluctuations has a finite variance but instead assumes that it has a power-law tail distribution decreasing as $|x|^{-(\alpha+1)}$ with $0<\alpha \leq 2$. In that case, the density of individuals tends towards a stable Lévy distribution with exponent $\alpha$ [16], which is solution of a diffusion equation of fractional order $\alpha$ (see [28] for details). Here we restrict ourselves to $1<\alpha \leq 2$. It is important to note that when $\alpha=2$, the Lévy distribution reduces to a Normal distribution and the corresponding differential equation is the classical second-order diffusion equation. Eqs. (1)-(3) are therefore a generalization of a standard SIR model describing the spatial spread of an epidemic, as described for instance in [29], and reduce to such a model when $\alpha=2$. With power-law tail distribution decreasing as $|x|^{-(\alpha+1)}$, one can see that the probability of large displacements (called Lévy flights) increases as the value of $\alpha$ decreases. Fig. 1 illustrates the random displacements of a single individual following a Lévy motion for different values of the exponent $\alpha$.

Before moving to the analysis of the solutions of Eqs. (1)-(2), let us first recast them in non-dimensional form. Following Murray [29], we introduce the following dimensionless variables:

$$
I^{*}=I / \mathcal{S}, \quad S^{*}=S / \mathcal{S}, \quad x^{*}=\left(\frac{r \mathcal{S}}{K_{\alpha}}\right)^{1 / \alpha} x, \quad t^{*}=r \mathcal{S} t, \quad \lambda=\frac{a}{r \mathcal{S}},
$$



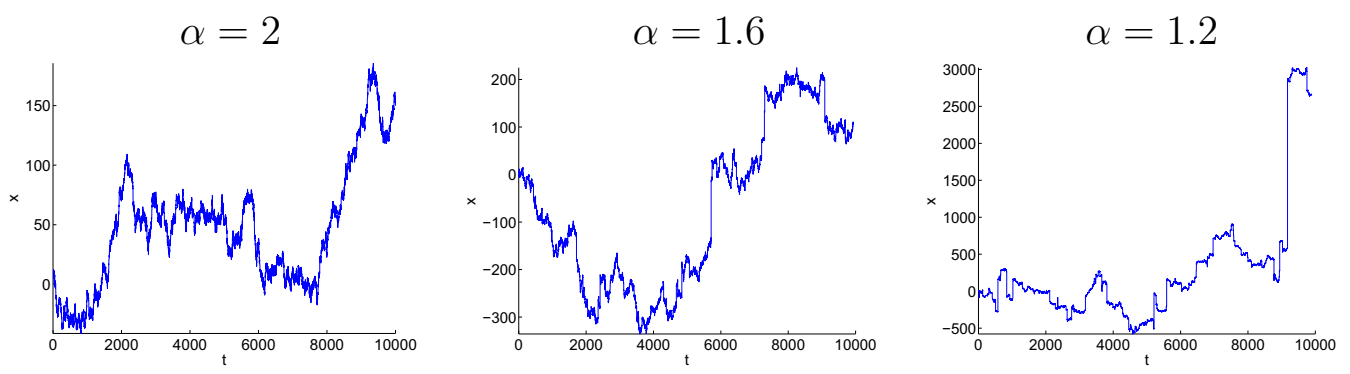

Figure 1: Trajectories of a random walker following a Lévy motion with different values of the exponent $\alpha$. Brownian motion is recovered when $\alpha=2$. Note the changes in the range of the vertical axis (position) while the horizontal axis (time) remains the same.

which allow us to rewrite Eqs. (1)-(2) as follows:

$$
\begin{aligned}
& \frac{\partial S}{\partial t}=-I S+{ }_{-\infty} D_{x}^{\alpha} S \\
& \frac{\partial I}{\partial t}=I S-\lambda I+-_{-\infty} D_{x}^{\alpha} I,
\end{aligned}
$$

where we have dropped the "*". As mentionned by Murray [29], the dimensionless parameter $\lambda$ is the ratio between the contagious time of the disease and the life expectancy. It is a key parameter that controls the development of the epidemic wave.

\section{Theoretical Analysis}

In this section, we investigate the spatial spread of an epidemic wave of infectives into a uniform population of susceptibles. In order to highlight the impact of the fractional-order diffusion operator ${ }_{-\infty} D_{x}^{\alpha}$ on the dynamics of the epidemic wave, we consider both left- and right-propagating fronts. The following analysis shares similarities with the one performed by del Castillo Negrete et al. [8] for the fractional-order Fisher-Kolmogorov equation. For that equation and by using the same left-sided diffusion operator as here, they found that left-propagating fronts have a self-similar profile and move at a constant speed, while right-propagating fronts are accelerated and exhibit a algebraic (power-law) decay of the tail. 


\subsection{Left-propagating front}

For such a front, we look for a self-similar travelling-wave solution by setting

$$
I(x, t)=I(z), \quad S(x, t)=S(z), \quad z=x+c t,
$$

where $c$ is the unknown positive wave speed. Note that the "+" in the definition of $z$ indicates that the wave is travelling to the left. Furthermore, we replace $S(z)$ by $1-s(z)$, where $s(z)$ is the deviation with respect to the initial susceptibles density. Eqs. (5)-(6) can then be expressed as:

$$
\begin{aligned}
-c \frac{\mathrm{d} s}{\mathrm{~d} z} & =-I(1-s)-{ }_{-\infty} D_{z}^{\alpha} s, \\
c \frac{\mathrm{d} I}{\mathrm{~d} z} & =I(1-s)-\lambda I+{ }_{-\infty} D_{z}^{\alpha} I .
\end{aligned}
$$

It can easily be seen that the Jacobian of the non-linear reaction term in Eqs. (7)-(8) only has real eigenvalues that reach their maximum value when $s=0$. The front is thus driven by the region just ahead of it or, in other words, it is pulled by its leading edge. Such a front is thus referred to as a pulled front and its speed can be derived by performing a linear analysis in the leading edge region [5]. In our case, the leading edge is where $I \rightarrow 0$ and $s \rightarrow 0$ (see Fig. 2a for an illustration) and linearizing the equations in that region results in the following system:

$$
\begin{aligned}
-c \frac{\mathrm{d} s}{\mathrm{~d} z} & =-I-{ }_{-\infty} D_{z}^{\alpha} s \\
c \frac{\mathrm{d} I}{\mathrm{~d} z} & =(1-\lambda) I+{ }_{-\infty} D_{z}^{\alpha} I .
\end{aligned}
$$

Assuming a solution of the form $(S(z), I(z))=(\hat{S}, \hat{I}) e^{\kappa z}$, where $\kappa$ is a parameter depending on the wave speed, and using the fact that ${ }_{-\infty} D_{z}^{\alpha} e^{\kappa z}=$ $\kappa^{\alpha} e^{\kappa z}$, one obtains the following system:

$$
\left(\begin{array}{cc}
c \kappa-\kappa^{\alpha} & -1 \\
0 & c \kappa-\kappa^{\alpha}-(1-\lambda)
\end{array}\right)\left(\begin{array}{c}
\hat{S} \\
\hat{I}
\end{array}\right)=\left(\begin{array}{l}
0 \\
0
\end{array}\right) .
$$

A non-trivial solution can only be obtained if

$$
\left(c \kappa-\kappa^{\alpha}\right)^{2}-(1-\lambda)\left(c \kappa-\kappa^{\alpha}\right)=0,
$$


which results in the following dispersion relations:

$$
\begin{aligned}
& c(\kappa)=\kappa^{\alpha-1}, \\
& c(\kappa)=\kappa^{\alpha-1}+\frac{1-\lambda}{\kappa} .
\end{aligned}
$$

As for the Fisher-Kolmogorov equation, we expect the front to propagate at the minimum wave speed for a "sufficiently steep" initial condition. Relation (11) is thus discarded as it would result in a non-moving front. By minimizing relation (12) with respect to $\kappa$, one finds the minimum value of the left-propagating front speed $c_{\min }$ and the corresponding exponential decay rate $\kappa_{\min }$ to be:

$$
c_{\text {min }}=\alpha\left(\frac{1-\lambda}{\alpha-1}\right)^{\frac{\alpha-1}{\alpha}}, \quad \kappa_{\min }=\left(\frac{1-\lambda}{\alpha-1}\right)^{1 / \alpha} .
$$

The former reduces to the classical result, $c=2 \sqrt{1-\lambda}$, when $\alpha=2$. Since we are assuming that $1<\alpha \leq 2, c_{\min }$ and $\kappa_{\min }$ are both well defined. The existence of a left-propagating front in the fractional diffusion case is still conditional on $\lambda<1$. Note that the more general method proposed by van Saarloos et al. $[14,38]$ leads to the same results (see Appendix A for details).

The value of the front speed can also be obtained by using a more heuristic argument. Indeed, at the leading edge, the linearization of Eq. (6) is similar to the linearization of the fractional-order Fisher-Kolmogorov equation. The same approach as in [8] can thus be used to derive the speed of the $I$ front, which leads to Eq. (13). Moreover, it is easily seen that without infectives there is no susceptibles front as the reaction term in Eq. (5) would vanish. This means that the susceptibles solution is driven by the infectives solution and thus both fronts travel at the same speed.

\subsection{Right-propagating front}

That case is a bit more complex as the velocity of the traveling wave is no more constant. One can thus not look for a self-similar solution. In what follows, we pursue the same approach as the one used by del Castillo Negrete et al. [8] for the fractional-order Fisher-Kolmogorov equation. Starting from Eq. (10), we can again linearize it at the leading edge, i.e. around $S=1$ (see Fig. 4a for an illustration), and obtain:

$$
\frac{\partial I}{\partial t}=(1-\lambda) I+{ }_{-\infty} D_{x}^{\alpha} I
$$


Since the term $(1-\lambda) I$ is responsible for the exponential growth or decay of the solution, one can assume a solution of the form $I(x, t)=e^{(1-\lambda) t} \psi(x, t)$. Substituting this solution into (20), one finds a differential equation for $\psi$ :

$$
\frac{\partial \psi}{\partial t}={ }_{-\infty} D_{x}^{\alpha} \psi
$$

with $\psi(x, t=0)=I(x, t=0)=I_{0}(x)$, the initial condition for the infectives population.

The general solution of Eq. (15) can be expressed as

$$
\psi(x, t)=\int_{-\infty}^{\infty} G\left(x-x_{0}, t\right) I_{0}\left(x_{0}\right) \mathrm{d} x_{0},
$$

where $G(x, t)=\frac{1}{t^{1 / \alpha}} p_{\alpha}\left(\frac{x}{t^{1 / \alpha}}\right)$ is the Green function of Eq. (15) and $p_{\alpha}(\eta)=$ $\frac{1}{2 \pi} \int_{-\infty}^{\infty} e^{i k \eta} e^{(i k)^{\alpha}} \mathrm{d} k$ is a skewed Lévy distribution with exponent $\alpha[26,16]$. By considering a localized initial condition of the form $I_{0}(x<-l)=0$; $I_{0}(-l \leq x<0)=A$ and $I_{0}(x \geq 0)=A e^{-\kappa x}-$ where $l$ and $A$ are arbitrary positive and non-zero constants, respectively - one finds the relation

$$
\psi(x, t)=A \int_{x t^{-1 / \alpha}}^{\infty} p_{\alpha}(\eta) \mathrm{d} \eta+A e^{-\kappa x} \int_{-\infty}^{x t^{-1 / \alpha}} p_{\alpha}(\eta) e^{\kappa t^{1 / \alpha} \eta} \mathrm{d} \eta
$$

As we look for the asymptotic behaviour, we consider solutions for large, fixed $t$ and $x \rightarrow \infty$. In that case, we can use the asymptotic behaviour of the Lévy distributions, i.e. $p_{\alpha}(\eta) \sim \eta^{-(\alpha+1)}$ as $\eta \rightarrow \infty$. After some algebraic manipulations (see Appendix B for details), one finds the asymptotic behaviour of $I$ :

$I(x, t)=A t e^{(1-\lambda) t}\left(\left(l+\frac{1}{\kappa}\right) x^{-(\alpha+1)}+\frac{1+\alpha}{\kappa t^{1+1 / \alpha}} \int_{-\infty}^{x t^{-1 / \alpha}} \frac{e^{\kappa\left(t^{-1 / \alpha} \eta-x\right)}}{\eta^{\alpha+2}} \mathrm{~d} \eta+\ldots\right)$.

The right-propagating front thus asymptotically decays as

$$
I(x, t) \sim t e^{(1-\lambda) t} x^{-(\alpha+1)},
$$

which highlights an algebraic (power-law) decaying tail that totally differs from the exponential decay observed for the left-moving front and from classical results obtained with second-order diffusion operators.

The solution for the susceptibles population is obtained by assuming again that $S(x, t)=1-s(x, t)$ where $s(x, t) \ll 1$. The following expression can 
then be derived: $s(x, t)=\frac{1}{1-\lambda} e^{(1-\lambda) t} \psi(x, t)$, which leads to the asymptotic behaviour:

$$
1-S(x, t) \sim t e^{(1-\lambda) t} x^{-(\alpha+1)} .
$$

Just as for the left-propagating front, the susceptibles solution is entirely driven by the infectives solution, i.e. in the absence of infectives, there is no susceptibles front. As a result, the decay rate of the $S$ front is the same as for the $I$ front whatever the initial condition for $S$. In other words, even if $S$ is initially nonzero over the entire domain, its decay rate will be of the form $x^{-(\alpha+1)}$ rather than $x^{-\alpha}$, provided that $I$ is initially confined. However, if $I$ is not initially confined but greater than zero over the entire domain, the decay rate for both solutions will be of the form $x^{-\alpha}$ (see Appendix B).

The asymptotic front speed can be derived from either Eq. (17) or (18) by computing the Lagrangian trajectory of a point at the leading edge of the front. For instance, if we consider a point with a fixed value $\hat{S} \approx 1$, its position $\hat{x}=x(t, \hat{S})$ can be expressed as

$$
\hat{x} \sim(1-\hat{S})^{-1 /(\alpha+1)} t^{1 /(\alpha+1)} e^{\frac{1-\lambda}{\alpha+1} t},
$$

and the front speed, $c_{R}(t)=\frac{\mathrm{d} \hat{x}}{\mathrm{~d} t}$, therefore reads

$$
\begin{aligned}
c_{R}(t) & \sim(1-\hat{S})^{-1 /(\alpha+1)} \frac{t^{1 /(\alpha+1)}}{\alpha+1} e^{\frac{1-\lambda}{\alpha+1} t}\left(\frac{1}{t}+1-\lambda\right), \\
& \sim(1-\lambda) e^{\frac{1-\lambda}{\alpha+1} t},
\end{aligned}
$$

for large values of $t$. This highlights the exponential acceleration of rightpropagating fronts.

\section{Numerical examples}

In this section, we present some numerical simulations that illustrate the theoretical results derived in the previous section. The model Eqs. (1)-(2) are solved on a finite domain $[0, L]$, where $L>0$, and the fractional-order derivative is thus defined as follows:

$$
{ }_{0} D_{x}^{\alpha} f(x)=\frac{1}{\Gamma(2-\alpha)} \frac{\partial^{2}}{\partial x^{2}} \int_{0}^{x} \frac{f(y)}{(x-y)^{\alpha-1}} \mathrm{~d} y,
$$

where $1<\alpha \leq 2$ and $0 \leq x \leq L$, since model variables are now only defined on $[0, L]$. Solving the model equations on a finite domain has an impact on 
the behavior of the solution as fractional-order derivatives take into account the global aspect of the solution, which is obviously modified when truncating the domain. One can still take into account the effect of an infinite domain by using the following equations:

$$
\begin{aligned}
& \frac{\partial S}{\partial t}=-I S+{ }_{0} D_{x}^{\alpha}\left(S-S(0)-S^{\prime}(0)\right), \\
& \frac{\partial I}{\partial t}=I S-\lambda I+{ }_{0} D_{x}^{\alpha}\left(I-I(0)-I^{\prime}(0)\right),
\end{aligned}
$$

where $S(0)=S(x=0, t)$ and $S^{\prime}(0)=S^{\prime}(x=0, t)$, and the same for $I$. In Eqs. (19)-(20), the fractional-order derivative is now a so-called Caputo fractional derivative that can be defined as follows:

$$
\begin{aligned}
{ }_{0}^{C} D_{x}^{\alpha} f(x) & =\frac{1}{\Gamma(2-\alpha)} \int_{0}^{x} \frac{f^{\prime \prime}(y)}{(x-y)^{\alpha-1}} \mathrm{~d} y, \\
& ={ }_{0} D_{x}^{\alpha}\left(f(x)-f(0)-f^{\prime}(0)\right) .
\end{aligned}
$$

By using some standard properties of the Caputo derivative, it can be shown that Eqs. (19)-(20) are equivalent to Eqs. (5)-(6) if one assumes constant density values for $x \leq 0$, i.e. $I(x<0, t)=I(0)$ and $S(x<0, t)=S(0)$. One can then represent the effect of an infinite reservoir of susceptibles and/or infectives at the left-hand side of the domain by selecting an initial condition which is such that $S(0)>0$ and/or $I(0)>0$.

Eqs. (19)-(20) are discretized with a continuous piecewise-linear finiteelement scheme on a uniform grid whose resolution is equal to $L / 500$. The finite element scheme is based on a Galerkin formulation that allows one to integrate the fractional-order diffusion term by parts and impose a vanishing fractional-order flux on the left boundary (see [20] for details). A zero-slope boundary condition is imposed on the right boundary. The diffusion term being entirely left-sided, the right boundary condition only has a local impact on the solution. The use of a zero-slope boundary condition allows the right-propagating front to smoothly leave the domain. Time integration is computed with a third-order Adams-Bashforth scheme.

The following initial conditions are used for both left- and right-propagating fronts:

$$
\begin{aligned}
& S_{0}(x)=1-h_{S}\left(1 \pm \tanh \left(\frac{x-x_{0}}{w_{S} L}\right)\right) \\
& I_{0}(x)=h_{I} \exp \left(-\frac{\left(x-x_{0}\right)^{2}}{w_{I} L^{2}}\right)
\end{aligned}
$$


where $w_{S}=0.009, h_{S}=0.37, w_{I}=0.0003$ and $h_{I}=0.14$. For a leftpropagating front it takes the "+" sign and $x_{0}=9 L / 10$, whereas for a right-propagating front, $S_{0}(x)$ takes the "-" sign and $x_{0}=L / 10$ or 0 . The dimensionless domain length is set to $L=10^{5}$ for a right-propagating front and $L=60$ for a left-propagating front. For all simulations, $\lambda=0.5$.

The time evolution of a left-propagating front is shown in Fig. 2. The top panel shows the evolution of both the susceptibles and infectives densities. The middle and bottom panels show a close-up view on the susceptibles and infectives density tail decay. The duration of the simulation is set to 30 dimensionless time units and $\alpha=1.2$. As expected, both the susceptibles and infectives fronts propagate at the same speed and exhibit the same exponentially decaying tail, i.e. $1-S$ and $I \sim \exp \left[\left(\frac{1-\lambda}{\alpha-1}\right)^{1 / \alpha} x\right]$. The front speed has been numerically estimated by computing the instantaneous speed of a point with density $S=0.99$ (Fig. 3). The resulting time series highlights the convergence of the front speed towards the minimum speed $c_{\min }=1.398$, obtained from Eq. (13).

The time evolution of a right-propagating front is shown in Fig. 4. We first consider a localized initial condition for the infectives by taking $x_{0}=$ $L / 10$. In that case, $I(0)=0$ and there is no reservoir of infectives at the lefthad side of the computational domain. However, $S(0)>0$ and there is thus an infinite reservoir of susceptibles at the left-hand side. Such a situation is quite realistic as an epidemic usually starts with a confined population of infective individuals in contact with a broad population of susceptible individuals. The simulation duration and the value of $\alpha$ are the same as for the left-propagating front. As expected, both the susceptibles and infectives fronts accelerate and develop the same algebraic decaying tail, i.e. $1-S$ and $I \sim x^{-(\alpha+1)}$. After an initial adjustment, the acceleration of the front leads to a rapid spread of the epidemic over the entire domain. It should be noted that the length of the domain is equal to $10^{5}$ as compared to 60 for the left-progagating front simulation whereas the simulation duration remains the same. It should also be noted that infinite reservoir of susceptibles does not influence the rate of decay. The epidemic being driven by the infectives and not by the susceptibles density, the front dynamics entirely depends on the infectives density. Actually, even if there was no diffusion term in the $S$ equation and thus if susceptible individuals were not moving, there would still be the same epidemic front.

The acceleration of the right-propagating front is highlighted in the space- 

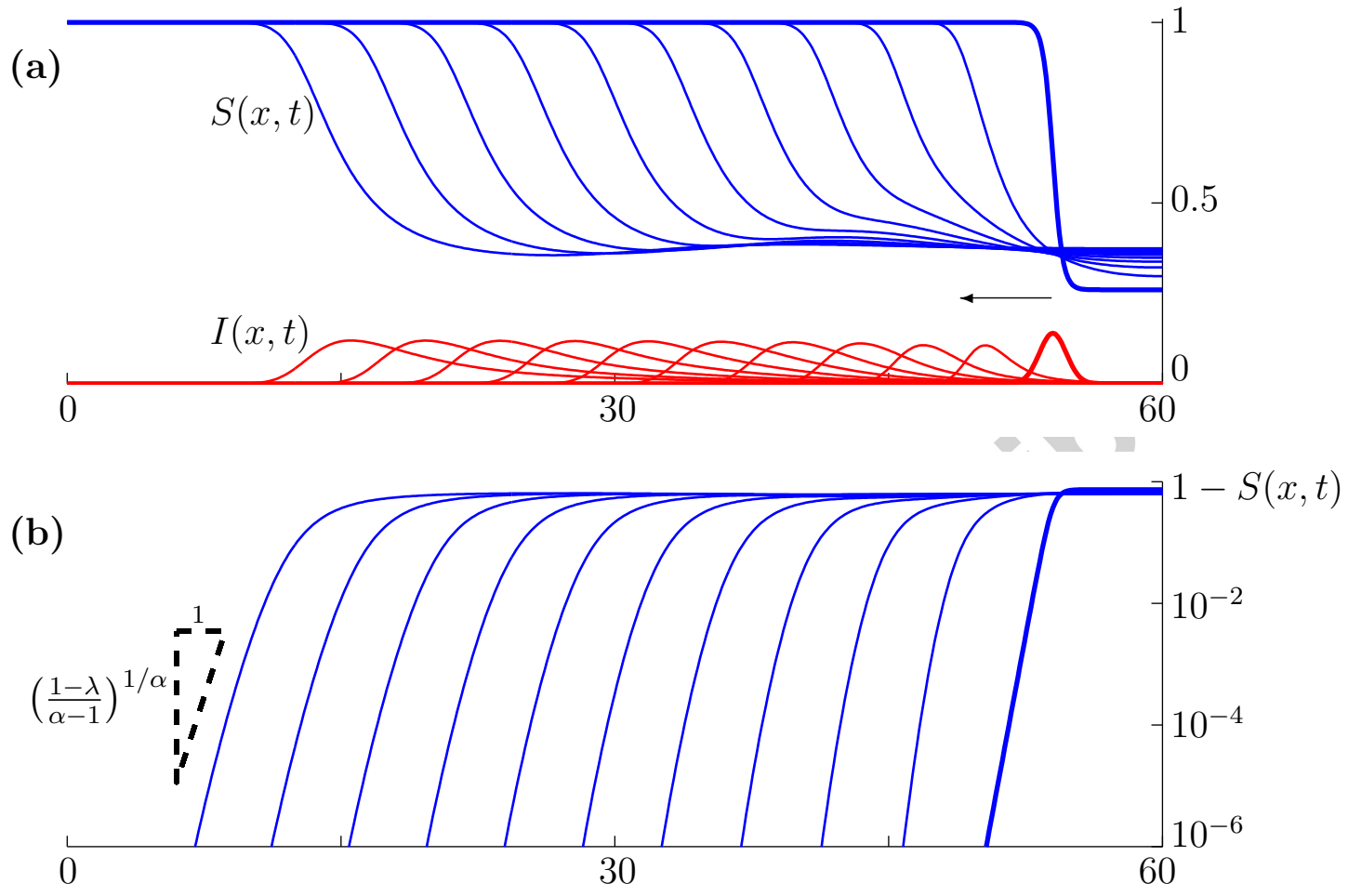

(c)

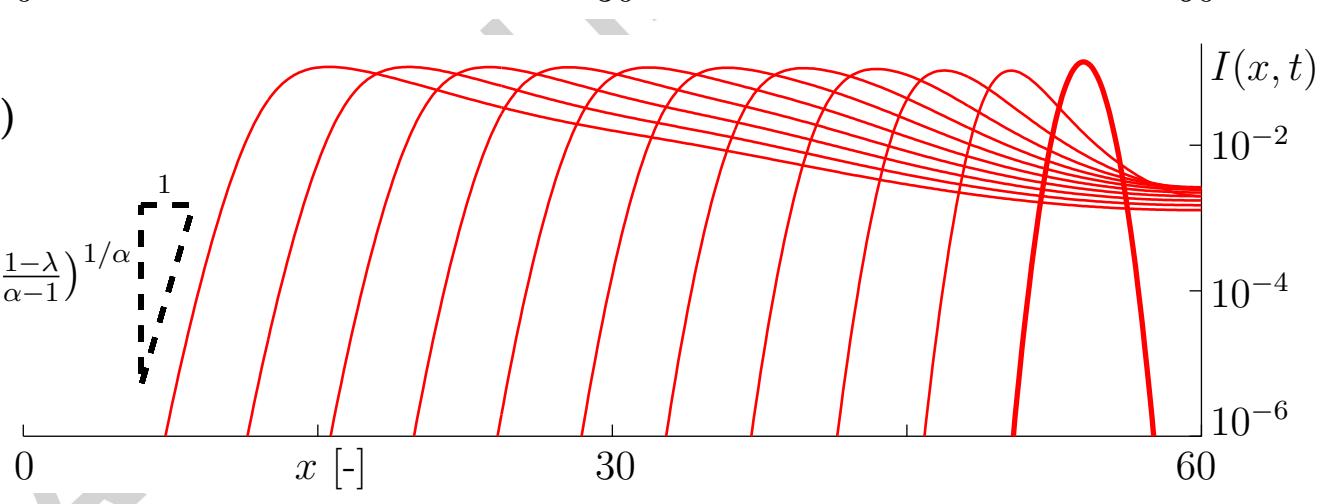

Figure 2: (a) Left-propagating front profiles for the susceptibles and infectives densities at different time instants obtained by solving Eqs. (19)-(20) with $\alpha=1.2$. The arrow indicates the direction of propagation of the front. (b) and (c) Close-up views of the susceptibles and infectives densities, respectively, highlighting the same exponentially decaying tail for both fronts, i.e. $1-S$ and $I \sim \exp \left[\left(\frac{1-\lambda}{\alpha-1}\right)^{1 / \alpha} x\right]$, and the same front speeds. The simulation duration is set to 30 and the time interval between front profiles is set to 3 . 


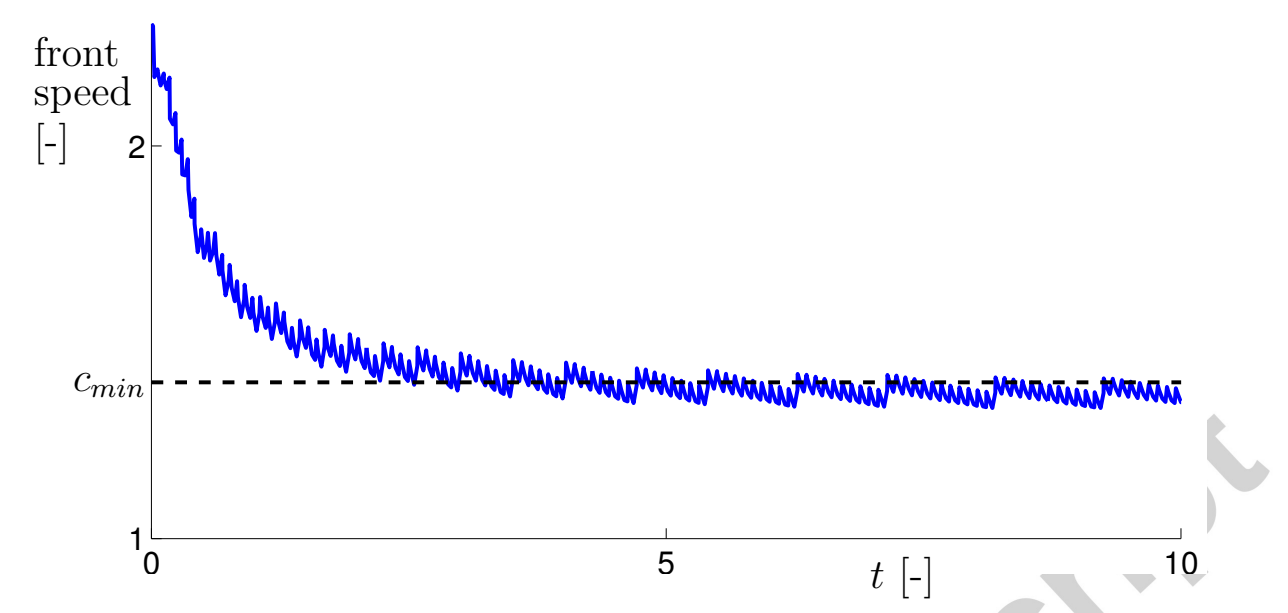

Figure 3: Time evolution of the left-propagating front instantaneous speed, taken as the time derivative of the Lagrangian trajectory $x_{f}(t ; S=0.99)$, for $\alpha=1.2$. As expected, the front speed converges towards the minimum front speed value $c_{\text {min }} \approx 1.398$.

time diagram shown in Fig. 5. It shows the evolution of the position of the front leading edge defined as the Lagrangian trajectory $x_{f}(t)=x(t ; S=0.99)$ that corresponds to the position of a point in the front with density $S=0.99$. The simulation duration is set to 60 . It can be seen that the front eventually accelerates as soon as $\alpha<2$. The smaller the value of $\alpha$, the sooner the acceleration takes place. Fig. 5 also shows that the numerical results are in good agreement with the asymptotic expansion, i.e. $1-S \sim x^{-(\alpha+1)} e^{(1-\lambda) t}$.

Although that might not be very realistic, one can still represent the effect of an infinite reservoir of infective individuals located at the left-hand side of the domain by considering an initial solution that does not vanish at $x=0$. This is achieved by taking $x_{0}=0$ in the initial conditions (21)-(22) such that $I(0)>0$ and $S(0)>0$. In that case, the front is expected to exhibit an algebraic decay rate of order $-\alpha$ rather than $-(\alpha+1)$ (see Appendix B). Fig. 6 shows that such an asymptotic behavior is indeed observed as $1-S$ and $I \sim x^{-\alpha}$. These results are similar to those obtained by del Castillo Negrete et al. [8] for the fractional-order Fisher-Kolmogorov model for which they had also considered an initial condition that did not vanish at $x=0$. 

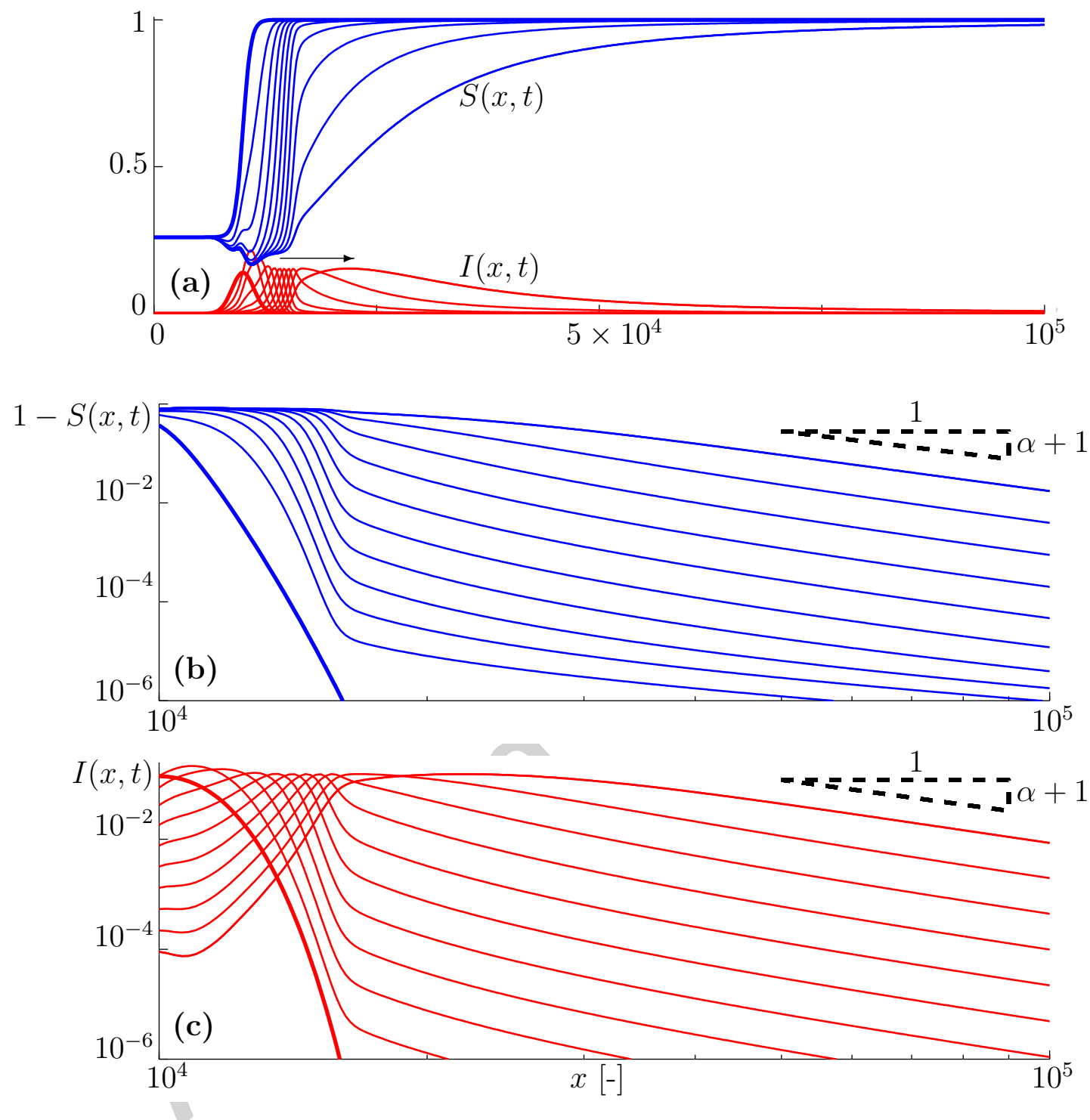

Figure 4: (a) Right-propagating front profiles for the susceptibles and infectives densities at different time instants obtained by solving Eqs. (19)-(20) with $\alpha=1.2$. The arrow indicates the direction of propagation of the front. (b) and (c) Close-up views of the susceptibles and infectives densities, respectively, highlighting the same power-law decaying tail for both fronts, i.e. $1-S$ and $I \sim x^{-(\alpha+1)}$. The simulation duration is set to 30 and the time interval between front profiles is set to 3 . 


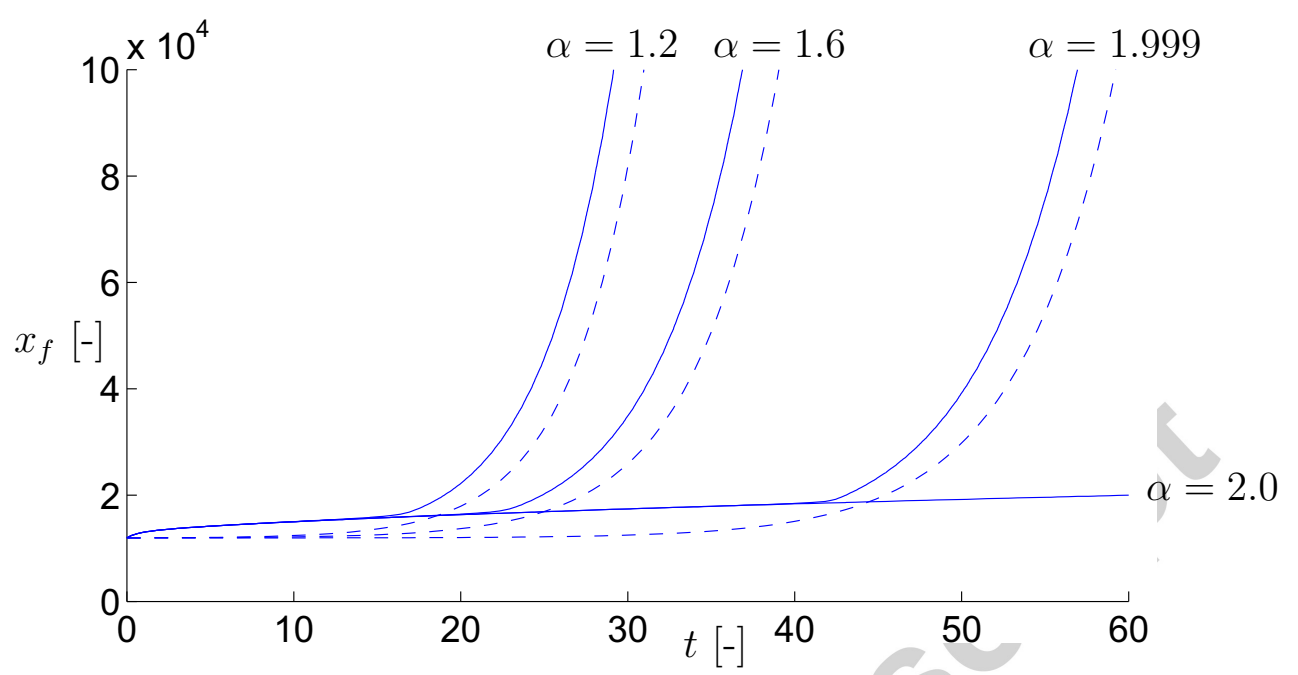

Figure 5: Time evolution of the epidemic front leading edge position, taken as the Lagrangian trajectory $x_{f}(t ; S=0.99)$, for different values of $\alpha$. As soon as $\alpha<2$, the front eventually accelerates leading to long-range spreading of the epidemic. The dashed line corresponds to the analytical scaling result $1-S \sim x^{-(\alpha+1)} e^{(1-\lambda) t}$.

\section{Conclusions}

In this paper, we have proposed a fractional-order reaction-diffusion model to study the dynamics of epidemics in systems characterized by asymmetric Lévy flights with an exponent $1<\alpha \leq 2$. We have considered a totally skewed, left-sided diffusion operator and studied its impact on left- and right-propagating fronts. On the one hand, numerical and analytical results show that left-propagating fronts move at a constant velocity and have an exponentially decaying tail. The classical results for the front velocity are recovered when $\alpha=2$. On the other hand, right-propagating fronts accelerate exponentially and exhibit a power-law decaying tail. Both the infectives and susceptibles fronts have the same dynamics which is entirely driven by the infectives density. For an initially localized infectives density profile, the decay is of the form $\sim x^{-(\alpha+1)}$ while an infinitely-wide initial profile leads to a decay of the form $\sim x^{-\alpha}$ for both solutions. Obviously, for a symmetric fractional-order diffusion operator, both the left- and right-propagating fronts accelerate and have a power-law decaying tail.

Our results show that a fractional-order reaction-diffusion epidemics model is able to represent the superdiffusive effect due to Lévy-flight mobility pat- 

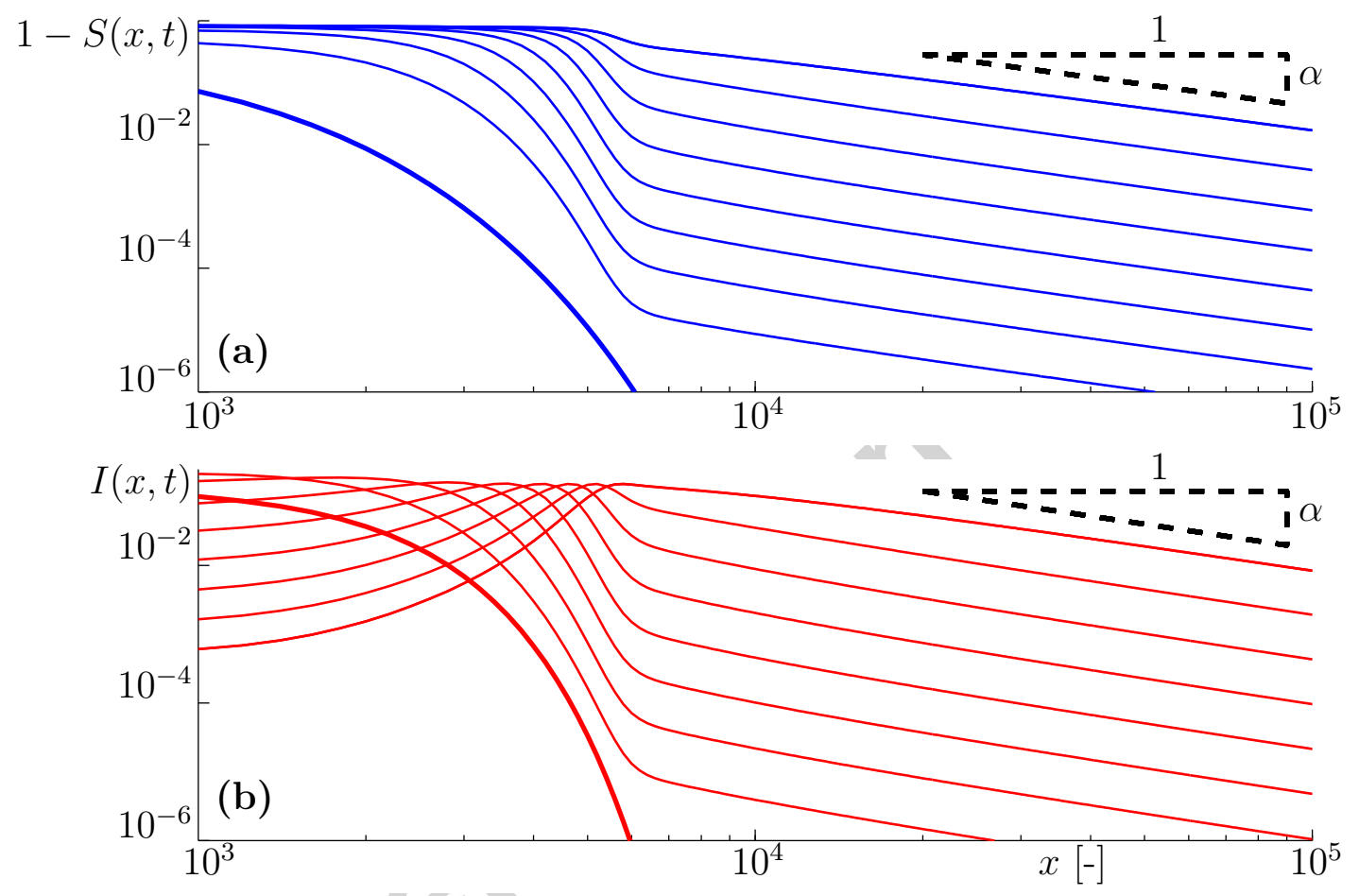

Figure 6: Right-propagating front profiles for the (a) susceptibles and (b) infectives density at different time instants obtained by solving Eqs. (19)-(20) with $\alpha=1.2$ and taking $x_{0}=0$ in the initial conditions (21)-(22). The power-law decaying tail is the same for both fronts, i.e. $1-S$ and $I \sim x^{-\alpha}$. The simulation duration is set to 24 and the time interval between front profiles is set to 3 . 
terns in the population. The superdiffusion of the infective population leads to a significantly increased overall reaction rate as both populations meet each other more often. As a result, an epidemic spreads much faster than predicted by classical Gaussian models. Our observations suggest that fractional-order reaction-diffusion models are better suited to represent modern epidemics and also highlight that the eradication or even containment of such epidemics is a daunting task.

The model could be further improved by taking into account non-Markovian and truncated Lévy processes. Brockmann et al. [2] have shown that the dispersion of bank notes is not only non-Gaussian but also non-Markovian. The non-Markovian effect and the associated subdiffusion can be included in our model by replacing the first-order time derivative with a fractionalorder time derivative of order less than 1 (see [28] for details). Numerical methods have recently been proposed to discretize the space-time fractional diffusion equation [34, 21] and could be applied in a non-Gaussian and nonMarkovian epidemics models. Furthermore, the study by Gonzalez et al. on human mobility patterns derived from mobile phone data suggests that these patterns follow a truncated Lévy-flight motion [18]. The effect of truncation on superdiffusive fronts propagation has been studied by del Castillo Negrete

[7] for a Fisher-Kolmogorov model and should be considered for epidemics models as well.

\section{Acknowledgements}

Eric Deleersnijder is a Research associate with the Belgian National Fund for Scientific Research (FNRS).

\section{Appendix A. Alternative derivation of the left-moving front speed}

In this section, we present a more general derivation of Eqs. (13) based on the approach proposed by van Saarloos et al. [14, 38]. In those studies, the authors define an asymptotic spreading speed, that they denote $v^{*}$, towards which the front eventually converges if it evolves from a sufficiently steep initial state. That speed is obtained by linearizing the model equations about the unstable state ( $S=1$ and $I=0$ in our case). More specifically, $v^{*} \in \mathbb{R}$ is given by the largest "dynamically relevant" solution of the saddle points 
344

equations

$$
\left\{\begin{aligned}
0 & =\mathcal{S}\left(k^{*}, \omega^{*}\right), \\
v^{*} & =\left.\frac{\mathrm{d} \omega(k)}{\mathrm{d} k_{k}}\right|_{k^{*}}, \\
v^{*} & =\frac{\operatorname{Im} \omega^{*}}{\operatorname{Im} k^{*}},
\end{aligned}\right.
$$

solved for $\left(k^{*}, \omega^{*}, v^{*}\right)$ where $\mathcal{S}(k, \omega)=0$ is the characteristic equation obtained by assuming a Fourier mode solution of the form $e^{i(k x-\omega t)}$ ( $k$ and $\omega \in \mathbb{C}$ ) in the linearization about the unstable state ahead of the front.

In our case, the characteristic equation reads:

$$
\mathcal{S}(k, \omega)=\left(-i \omega-(i k)^{\alpha}\right)^{2}-(1-\lambda)\left(-i \omega-(i k)^{\alpha}\right)=0
$$

and has two solutions: $\omega=i(i k)^{\alpha}$ and $\omega=i(i k)^{\alpha}+i(1-\lambda)$. The former is rejected as it leads to $v^{*}=0$. The latter corresponds to a non-oscillating, uniformly translating front if $k^{*}$ and $\omega^{*}$ are purely imaginary, which can only be achieved if $k^{*}=-i \kappa^{*}\left(\kappa^{*} \in \mathbb{R}^{+}\right)$. In that case,

$$
\begin{aligned}
v^{*} & =\left.\frac{\mathrm{d} \omega(k)}{\mathrm{d} k}\right|_{k^{*}}=-\alpha\left(\kappa^{*}\right)^{\alpha-1}, \\
& =\frac{\operatorname{Im} \omega^{*}}{\operatorname{Im} k^{*}}=\frac{\left(\kappa^{*}\right)^{\alpha}+(1-\lambda)}{-\kappa^{*}},
\end{aligned}
$$

which leads to

$$
v^{*}=-\alpha\left(\frac{1-\lambda}{\alpha-1}\right)^{\frac{\alpha-1}{\alpha}}, \quad \kappa^{*}=\left(\frac{1-\lambda}{\alpha-1}\right)^{1 / \alpha} .
$$

As expected, these correspond to Eqs. (13) and highlight that only a leftpropagating front can travel at a constant speed as $v^{*}=-c_{\min }<0$. Moreover, van Saarloos et al. have shown that the front speed algebraically relaxes towards that constant speed if the initial state is steeper than $e^{\kappa^{*} x}$.

\section{Appendix B. Asymptotic behaviour of the right-moving front}

In this section, we provide more details on the derivation of Eq. (17). Let us first consider the first integral in Eq. (16) and look at the limit for $x t^{-1 / \alpha} \rightarrow \infty$ with a fixed $t$. In that case, we can use the asymptotic behaviour 
362

of the Lévy distribution: $p_{\alpha}(\eta) \sim \eta^{-(\alpha+1)}$ to obtain:

$$
\begin{aligned}
\int_{x t^{-1 / \alpha}}^{(x+l) t^{-1 / \alpha}} p_{\alpha}(\eta) \mathrm{d} \eta & \sim \int_{x t^{-1 / \alpha}}^{(x+l) t^{-1 / \alpha}} \frac{1}{\eta^{\alpha+1}} \mathrm{~d} \eta \\
& =\frac{-1}{\alpha}\left[\frac{1}{\eta^{\alpha}}\right]_{x t^{-1 / \alpha}}^{(x+l) t^{-1 / \alpha}}, \\
& =\frac{-t}{\alpha} x^{-\alpha}\left(\left(1+\frac{l}{x}\right)^{-\alpha}-1\right), \\
& \sim t l x^{-(\alpha+1)},
\end{aligned}
$$

where we have used a first-order binomial-series approximation.

For the second integral, one needs to set a cutoff $\Omega$ with $1 \ll \Omega<x t^{-1 / \alpha}$, so that $p_{\alpha}(\eta)=\eta^{-(\alpha+1)}$ holds. Integration by parts then leads to

$$
\begin{aligned}
& e^{-\kappa x} \int_{-\infty}^{x t^{-1 / \alpha}} p_{\alpha}(\eta) e^{\kappa t^{1 / \alpha} \eta} \mathrm{d} \eta \\
& \sim e^{-\kappa x} \int_{\Omega}^{x t^{-1 / \alpha}} \frac{1}{\eta^{\alpha+1}} e^{\kappa t^{1 / \alpha} \eta} \mathrm{d} \eta \\
& =e^{-\kappa x}\left[\frac{1}{\eta^{\alpha+1}} \frac{e^{\kappa t^{1 / \alpha} \eta}}{\kappa t^{1 / \alpha}}\right]_{\eta=\Omega}^{\eta=x t^{-1 / \alpha}}-e^{-\kappa x} \int_{\Omega}^{x t^{-1 / \alpha}}-\frac{\alpha+1}{\eta^{\alpha+2}} \frac{e^{\kappa t^{-1 / \alpha} \eta}}{\kappa t^{1 / \alpha}} \mathrm{d} \eta \\
& \sim e^{-\kappa x}\left(\frac{1}{x^{\alpha+1} t^{-1-1 / \alpha}} \frac{e^{\kappa t^{1 / \alpha} x t^{-1 / \alpha}}}{\kappa t^{1 / \alpha}}\right)+t \frac{(1+\alpha)}{\kappa t^{1+1 / \alpha}} \int_{\Omega}^{x t^{-1 / \alpha}} \frac{e^{\kappa\left(t^{-1 / \alpha} \eta-x\right)}}{\eta^{\alpha+2}} \mathrm{~d} \eta, \\
& =t\left(\frac{x^{-(\alpha+1)}}{\kappa}+\frac{(1+\alpha)}{\kappa t^{1+1 / \alpha}} \int_{\Omega}^{x t^{-1 / \alpha}} \frac{e^{\kappa\left(t^{-1 / \alpha} \eta-x\right)}}{\eta^{\alpha+2}} \mathrm{~d} \eta\right) .
\end{aligned}
$$

In fact, the integral from $-\infty$ to $\Omega$ contains $p_{\alpha}(\eta)$ that decays exponentially at minus infinity. The exponential in the integrand is bounded on this domain. We can thus consider that the integral is bounded and not dependant on $x$. The integrand in the last expression is bounded by $\frac{1}{\eta^{2+\alpha}}$ when $x \rightarrow \infty$ and thus, the third term is at most of order $x^{-(\alpha+1)}$.

From (B.1) and (B.2), we see that the tail of the right-propagating front behaves like $\psi \sim t x^{-(\alpha+1)}$ for large values of $x$ and $t$. Note that this behaviour is preserved in the limit where $l \rightarrow 0$. However, in the limit where $l \rightarrow \infty$, the asymptotic behaviour becomes $\psi \sim t x^{-\alpha}$. That case would represent the 
effect of an infinite initial reservoir of infective individuals on the epidemic front propagation. In the study by del Castillo Negrete et al. [8] for the fractional-order Fisher-Kolmogorov equation, non-localized initial conditions are considered, i.e. $l \rightarrow \infty$, and an algebraic decay rate of order $-\alpha$ is observed.

\section{References}

[1] Brockmann, D., 2009. Human mobility and spatial disease dynamics, in: Schuster, H.G. (Ed.), Reviews of Nonlinear Dynamics and Complexity, Wiley-VCH. pp. 1-24.

[2] Brockmann, D., Hufnagel, L., Geisel, T., 2006. The scaling laws of human travel. Nature 439, 462-5.

[3] Buchanan, M., 2008. Ecological modelling: The mathematical mirror to animal nature. Nature 453, 714-6.

[4] Cartea, A., del Castillo Negrete, D., 2007. Fractional diffusion models of option prices in markets with jumps. Physica A: Statistical Mechanics and its Applications 374, 749-63.

[5] Cencini, M., Lopez, C., Vergni, D., 2003. Reaction-diffusion systems: Front propagation and spatial structures, in: Vulpiani, A., Livi, R. (Eds.), The Kolmogorov legacy in physics, Springer, Berlin. pp. 187209.

[6] Chaves, A.S., 1998. A fractional diffusion equation to describe Lévy flights. Physics Letters A 239, 13-6.

[7] del Castillo Negrete, D., 2009. Truncation effects in superdiffusive front propagation with Lévy flights. Physical Review E 79, 1-10.

[8] del Castillo Negrete, D., Carreras, B. A., Lynch, V. E., 2003. Front dynamics in reaction-diffusion systems with Lévy flights: A fractional diffusion approach. Physical Review Letters 91.

[9] del Castillo Negrete, D., Carreras, B. A., Lynch, V. E., 2004. Fractional diffusion in plasma turbulence. Physics of Plasmas 11, 3854-64. 
[10] del Castillo Negrete, D., Carreras, B. A., Lynch, V. E., 2005. Nondiffusive transport in plasma turbulence: A fractional diffusion approach. Physical Review Letters 94.

[11] Deng, Z.Q., de Lima, J.L.M.P., de Lima, M.I.P., Singh, V.P., 2006. A fractional dispersion model for overland solute transport. Water Resources Research 42:W03416.

[12] Ding, Y., Ye, H., 2009. A fractional-order differential equation model of HIV infection of CD4+ T-cells. Mathematical and Computer Modelling $50,386-92$.

[13] Djordjević, V.D., Jarić, J., Fabry, B., Fredberg, J. J., Stamenović, D., 2003. Fractional derivatives embody essential features of cell rheological behavior. Annals of Biomedical Engineering 31, 692-9.

[14] Ebert, U., van Saarloos, W., 2000. Front propagation into unstable states: Universal algebraic convergence towards uniformly translating pulled fronts. Physica D 146, 1-99.

[15] Edwards, A.M., Phillips, R.A., Watkins, N.W., Freeman, M.P., Murphy, E.J., Afanasyev, V., Buldyrev, S.V., Da Luz, M.G.E., Raposo, E.P., Stanley, H.E., Viswanathan, G.M., 2007. Revisiting Lévy flight search patterns of wandering albatrosses, bumblebees and deer. Nature 449, 1044-8.

[16] Feller, W., 1971. An Introduction to Probability Theory and Its Applications, vol II. Wiley.

[17] Gnedenko, B., Kolmogorov, A., 1954. Limit Distributions for Sums of Independent Random Variables. Addison-Wesley.

[18] González, M.C., Hidalgo, C.A., Barabási, A.-L., 2008. Understanding individual human mobility patterns. Nature 453, 779-82.

[19] Gorenflo, R., Mainardi, F., Scalas, E., Raberto, R., 2010. Fractional calculus and continuous-time finance III: The diffusion limit, in: Kohlmann, M., Tang, S. (Eds.), Mathematical Finance, Birkhauser. pp. 171-80.

[20] Hanert, E., 2010a. A comparison of three Eulerian numerical methods for fractional-order transport models. Environmental Fluid Mechanics 10, 7-20. Doi:10.1007/s10652-009-9145-4. 
[21] Hanert, E., 2010b. On the numerical solution of space-time fractional diffusion models. Computers and Fluids in press. Doi:10.1016/j.compfluid.2010.08.010.

[22] Hufnagel, L., Brockmann, D., Geisel, T., 2004. Forecast and control of epidemics in a globalized world. Proceedings of the National Academy of Sciences of the United States of America 101, 15124-9.

[23] Humphries, N.E., Queiroz, N., Dyer, J.R.M., Pade, N.G., Musyl, M.K., Schaefer, K.M., Fuller, D.W., Brunnschweiler, J.M., Doyle, T.K., Houghton, J.D.R., Hays, G.C., Jones, C.S., Noble, L.R., Wearmouth, V.J., Southall, E.J., Sims, D.W., 2010. Environmental context explains Lévy and Brownian movement patterns of marine predators. Nature 465, 1066-9.

[24] Kim, S., Kavvas, M.L., 2006. Generalized Fick's law and fractional ADE for pollution transport in a river: Detailed derivation. Journal of Hydrologic Engineering 11, 80-3.

[25] Lévy, P., 1954. Théorie de l'Addition des Variables Aléatoires. GauthierVillars.

[26] Mainardi, F., Luchko, Y., Pagnini, G., 2001. The fundamental solution of the space-time fractional diffusion equation. Fractional Calculus and Applied Analysis 4, 153-92.

[27] Mainardi, F., Raberto, M., Gorenflo, R., Scalas, E., 2000. Fractional calculus and continuous-time finance II: The waiting-time distribution. Physica A: Statistical Mechanics and its Applications 287, 468-81.

[28] Metzler, R., Klafter, J., 2000. The random walk's guide to anomalous diffusion: A fractional dynamics approach. Physics Reports 339, 1-77.

[29] Murray, J., 2002. Mathematical Biology. II:Spatial Models and Biomedical Applications. Springer-Verlag, New York.

[30] Noble, J.V., 1974. Geographic and temporal development of plagues. Nature 250, 726-9.

[31] Oldham, K., Spagnier, J., 1974. The Fractional Calculus : Theory and Applications of Differentiation and Integration to Arbitrary Order. Academic Press. 
[32] Pachepsky, Y., Timlin, D., Rawls, W., 2003. Generalized Richards' equation to simulate water transport in unsaturated soils. Journal of Hydrology 272, 3-13.

[33] Podlubny, I., 1999. Fractional Differential Equations. Mathematics in Science and Engineering, Volume 198, Academic Press.

[34] Podlubny, I., Chechkin, A., Skovranek, T., Chen, Y., Jara, B.M.V., 2009. Matrix approach to discrete fractional calculus II: Partial fractional differential equations. Journal of Computational Physics 228, $3137-53$.

[35] Scalas, E., Gorenflo, R., Mainardi, F., 2000. Fractional calculus and continuous-time finance. Physica A: Statistical Mechanics and its Applications 284, 376-84.

[36] Sims, D.W., Southall, E.J., Humphries, N.E., Hays, G.C., Bradshaw, C.J.A., Pitchford, J.W., James, A., Ahmed, M.Z., Brierley, A.S., Hindell, M.A., Morritt, D., Musyl, M.K., Righton, D., Shepard, E.L.C., Wearmouth, V.J., Wilson, R.P., Witt, M.J., Metcalfe, J.D., 2008. Scaling laws of marine predator search behaviour. Nature 451, 1098-102.

[37] Small, M., D.M.. Walker, C.K.. Tse, 2007. Scale-free distribution of avian influenza outbreaks. Physical Review Letters 99.

[38] van Saarloos, W., 2003. Front propagation into unstable states. Physics Reports 386, 29-222.

[39] Viswanathan, G.M., Afanasyev, V., Buldyrev, S.V., Murphy, E.J., Prince, P.A., Stanley, H.E., 1996. Lévy flight search patterns of wandering albatrosses. Nature 381, 413-5.

[40] Viswanathan, G.M., Buldyrev, S.V., Havlin, S., Da Luz, M.G.E., Raposo, E.P., Stanley, H.E., 1999. Optimizing the success of random searches. Nature 401, 911-4. 